\title{
Analysis of screening failure of live donation for kidney transplantation: experience of a single medical center in central Taiwan
}

\author{
Cheng-Hsu Chen ${ }^{1}$, Ya-Yun Feng ${ }^{2}$, Kun-Yuan Chiu ${ }^{3}$, Cheng-Kuang Yang ${ }^{3}$, Yi-Syuan $\mathrm{Chen}^{2}$, Jia-Chian Wu², Ming-Ju Wu ${ }^{1}$ \\ ${ }^{1}$ Division of Nephrology, Department of Internal Medicine, Taichung Veterans General Hospital, Taichung City, Taiwan \\ ${ }^{2}$ Committee of Transplant Medicine, Taichung Veterans General Hospital, Taichung City, Taiwan \\ ${ }^{3}$ Division of Urology, Taichung Veterans General Hospital, Taichung City, Taiwan
}

Background: The organ shortage and long waiting list are global burden in treatment for end-stage renal disease (ESRD), thus, live kidney donation is currently available option with superior outcome and quick to improve quality of life. We hope to understand the causes of screening failure of live donation and to establish better standard operating procedures to enhance success of live kidney donation.

Methods: This study is retrospective evaluation conducted in Taichung Veterans General Hospital from January 2018 to September 2020. Subjects were recruited donor-recipient pairs pursuing living kidney transplantation (LKTx). The screening failure defined as donor-recipient pairs not to perform following LKTX after initial evaluation and analyzed the medical and non-medical factors of screening failure. We arbitrary divided 6 categories from recipients' aspects and seven categories from donors' aspects for analysis of screening failure for LKTX.

Results: A total of 203 donor-recipient pairs came for work-up process of LKTx at our transplant center, finally, only 48 pairs (23.6\%) underwent surgery and 155 pairs did not. The rate of screening failure was $76.4 \%$, and the affecting factors were 105 pairs (67.7\%) from recipients and 50 pairs $(32.3 \%)$ from donors. The most common concern from recipients were the unwilling of LKTx (fear of risk and reluctance of donor) (44.8\%), high immunological risk of rejection (20.0\%), and impaired cardiopulmonary function (9.5\%), on the contrary, the most common regards of potential donors were the uncertain wills of donation (24.0\%), underlying diseases (24.0\%), and suboptimal renal function (18.0\%).

Conclusions: In this study, we demonstrated the major causes of screening failure of LKTx in our institute. The unwilling of LKTX of recipients and uncertain wills of donation of donors were major conflict of success of pre-transplant survey. Further efforts should emphasize on how to break their concerns and barriers in order to benefit their quality of life and long-term survival. 